\title{
La estrategia de la Reina Roja: una propuesta metodológica para el análisis estadístico del periodismo informativo en Costa Rica
}

\author{
Alejandro Lizano Fernández \\ Bachiller y licenciado en Ciencias de la Comunicación Colectiva con énfasis en Periodismo, y bachiller en Filología Espa- \\ ñola por la Universidad de Costa Rica (ucr), donde ha hecho estudios de maestría académica en Lingüística. Labora como \\ editor de textos didácticos en el Programa de Producción de Material Didáctico Escrito (promade) de la Universidad Estatal \\ a Distancia (uned), institución en la que también se ha desempeñado como periodista en la Oficina Institucional de Merca- \\ deo y Comunicación. Dirección electrónica: alizano@uned.ac.cr
}

Recibido: 18 de Julio 2012 • Aceptado: 19 de Febrero 2013

\section{RESUMEN}

\begin{abstract}
El objetivo de este artículo es presentar ante la comunidad de investigadores en las áreas de comunicación colectiva y periodismo, una aproximación a la combinación novedosa de algunas metodologías (método de Kayser o muestreo no probabilístico de semanas compuestas o de semanas escalonadas, orden de rangos, gráficos de dispersión y coeficiente de correlación de Spearman) tomando como ejemplo un caso específico (periodismo informativo cultural, PIC), con el fin de evaluar su aplicabilidad en otros campos de la Información Periodística Especializada (IPE).
\end{abstract}

Palabras claves: periodismo cultural, Información Periodística Especializada, método de semanas escalonadas, método de orden de rangos y coeficiente de correlación de Spearman.

\section{ABSTRACT}

This paper presents to the Journalism and Mass Communication's researchers community an approach to the novel combination of some methodologies (Kayser Method or staggered week, Spearman's rank correlation coefficient, rank order, scatter plots), using the example of a specific case (cultural news), to assess their applicability to other fields of Specialized Journalism.

Key words: Arts Journalism, Specialized Journalism, staggered week method, rank-ordering method, and Spearman's rank correlation coefficient.

\section{Introducción}

Desde siempre, ha persistido una división artificial de separar el conocimiento científico del no científico a partir de los métodos de investigación utilizados y los objetos de estudio; tradicionalmente, se les ha dado un carácter más científico, precisamente, a las ciencias exactas, las ciencias naturales y las ciencias agronómicas; y un valor menos científico a las ciencias sociales, las letras y las humanidades.

Así, estas últimas han tenido siempre que "demostrar" su "cientificidad". Nos preguntamos, entonces, qué es una producción académica científica o si existe un método único. Pero realmente, se ha pasado por alto el valor y la validez de los estudios multidisciplinarios en el que un saber simbólico sirve de herramienta para otro 
campo de conocimiento. $\mathrm{Y}$ desde las ciencias sociales a veces nos es difícil innovar y traspasar las fronteras de lo común para darnos cuenta de que los fenómenos de la realidad social no están aislados.

Por lo tanto, es posible explorar, comprender, interpretar la realidad social desde diversas perspectivas y encontrar nuevos caminos para resolver un problema de estudio. Este artículo presenta el diseño de una investigación que reúne aportes de la estadística descriptiva y referencial como herramienta o método para un caso cuyo objeto de estudio se encuentra en las ciencias sociales.

Mediante la aplicación de algunas metodologías (método de Kayser o de semanas escalonadas, orden de rangos, gráficos de dispersión y coeficiente de correlación de Spearman), en esta investigación, específicamente, la metodología aplicada permite determinar, sobre todo, el nivel de correlación entre las noticias culturales de tres periódicos de circulación nacional en Costa Rica: La Nación, La República y La Prensa Libre en sus respectivas secciones: Cultura, Magazine y Abanico.

La preocupación social que está detrás del tema planteado es ampliar el espectro de posibilidades para abordar el fenómeno de la comunicación desde la academia y desde la práctica profesional, que, como bien apunta Jesús Martín-Barbero, tienden a escindirse en búsquedas dobles: por un lado, para resolver los procesos sociales de la comunicación y la producción social de sentido como tal y, por otro, la búsqueda desde los métodos o la hermenéutica. Pero en ambos casos subyace la necesidad de apartarse de la inmanencia del discurso universitario y que los resultados de la investigación sean accesibles a las personas dentro de la academia y fuera.

Se delimitó la periodización general de esta investigación a un año antes de este cambio de secciones y contenidos en La Nación, por ser justamente el referente: del 11 de febrero del 2004 al 10 de febrero del 2005. ¿Por qué no se ha seleccionado un período de análisis más reciente? En primer lugar, se ha escogido analizar La Nación por ser un referente obligatorio del periodismo nacional; como menciona Carlos Sandoval García, el Grupo Nación "se ha posicionado como uno de los grupos con mayor poder de decisión sobre la agenda de la industria cultural". (Sandoval, 2002: 51)

La versión anterior de la revista Viva (del 10 de febrero del 2004 hacia atrás) recogía las más variadas expresiones culturales; sin embargo, a partir del 11 de febrero del 2005 el Grupo Nación reestructuró el periódico: se creó Aldea Global, con una página interna: desde entonces, Arte \& Letras, que informa sobre pintura, escultura, arquitectura y patrimonio urbano; mientras la revista reformada de Viva, recoge noticias de teatro, danza y música, pero también espectáculos.

Así, en primer lugar, las noticias culturales se distribuyen en dos secciones diferentes. En segundo lugar, en la nueva revista Viva, a partir de la fecha citada, no hay una delimitación clara entre lo que se considera cultura y espectáculos. En ese sentido, Viva parece no tener una línea informativa clara sobre lo que es periodismo informativo cultural, no la presenta de manera ordenada ni coherente o tiende hacia el infotainment.

Aparte, si se hiciera una investigación exclusiva, o en comparación con otros medios, para Arte \& Letras, habría un sesgo en los resultados pues la muestra de dicha sección, en cuanto al contenido y la metodología (que posteriormente se explicará), estaría incompleta. En todo caso, habría que tomar en cuenta las noticias culturales de Viva (¿a partir de cuáles criterios?), a fin de suplir las faltantes en Arte \& Letras; pero sería muy complicado hacerlo si las noticias de Viva no tienen una cohesión temática cultural informativa clara, como se citó.

Adicionalmente, se eligieron las notas informativas de las revistas culturales de La República y La Prensa Libre por ser los otros dos periódicos nacionales de circulación diaria (además de La Nación en dicho momento histórico, desde luego) con secciones culturales bien definidas y diarias, lo cual no ocurre, por ejemplo, con rotativos como La Extra y La Teja (ambas por su corte sensacionalista y la última porque no existía durante el periodo en estudio), el semanario 
Universidad (por su periodicidad) ni Al Día (por su temática deportiva).

\section{Estado del objeto de estudio}

En cuanto a estudios en comunicación en lo que se aplica una metodología afín, se encuentra la tesis de Randall Sáenz Ugalde, quien en 1999 averigua la relación entre las categorías temáticas que constituyen la agenda editorial de los diarios La Nación, La República y La Prensa Libre, y su compatibilidad o conexión con la agenda del público. Se trata, pues, de una metodología poco aplicada en el campo de la comunicación colectiva, al menos localmente.

\section{Diseño de la investigación}

La investigación es de enfoque mixto pues involucra variables cualitativas y cuantitativas (que se especificarán posteriormente). Ambas variables permitirán conocer la realidad cotidiana, al tiempo que garantizan una mayor rigurosidad y exactitud de los resultados.

Se trata, además, de una investigación no experimental: se lleva a cabo sin manipular deliberadamente las variables; se basa en categorías, conceptos, contextos o sucesos que ya ocurrieron o que sucedieron sin la intervención directa del investigador (producción de noticias culturales). Presenta, además, un enfoque retrospectivo.

El diseño transeccional o transversal se realiza por observación de un momento único en el tiempo (noticias culturales publicadas en tres periódicos de circulación nacional desde el $11 \mathrm{de}$ febrero del 2004 hasta el 10 de febrero del 2005, como se explicó).

La investigación es de tendencia puesto que se verifican los cambios y las constantes (según las diversas variables prefijadas) en la producción de noticias durante el lapso predeterminado.

El estudio es correlacional pues describe las relaciones entre dos o más categorías, conceptos o variables en un momento determinado, que en este caso se trata de las agendas informativas culturales de cada medio.
La población o universo es "[...] la totalidad del fenómeno estudiado en donde las unidades de población poseen unas características comunes. [La población] se estudia y da origen a los datos de investigación" Tamayo y Tamayo (1994: 114). La población, en el caso en cuestión, es conformada por las noticias culturales de los tres medios escritos en las secciones citadas, publicadas de lunes a sábado entre el 11 de febrero del 2004 y el 10 de febrero del 2005.

La muestra es el "conjunto de operaciones [...] para estudiar la distribución de determinados caracteres en la totalidad de una población [...] partiendo de la observación [o análisis] de una fracción de la población considerada" (Tamayo y Tamayo, 1994: 115). Por ende, para seleccionar la muestra se aplica el Método de Kayser o de semanas escalonadas, el cual se detalla en la propuesta metodológica.

\section{Propuesta metodológica}

Para comparar las noticias culturales de las tres secciones culturales se aplicarán varias metodologías, consideradas las más adecuadas para este estudio estadístico descriptivo y referencial: método de Kayser o de semanas escalonadas, coeficiente de correlación de Spearman y gráficos de dispersión, además de análisis de contenido (que no se detallará conceptualmente pues correspondería a una fase posterior de la investigación).

\section{Método de Kayser}

En primer lugar, la selección de las fechas se definió por el Método de Kayser o muestreo no probabilístico de semanas compuestas o de semanas escalonadas. Con la metodología de la semana compuesta (Hansen, A., Cottle S., Negrine R. y Newbold C., 2002) se formula un calendario con los rotativos a partir del primer día de la primera semana del análisis (miércoles, en este caso); luego se avanza a la segunda semana y se toma el siguiente día en el orden: jueves; se continúa con la tercera semana y se toma el siguiente día (viernes). Así se procederá sucesivamente hasta llegar al último día del análisis en el último mes 
CUADRO 1

Ejemplo de muestreo no probabilística de semana compuesta (un mes)

\begin{tabular}{|c|c|c|c|c|c|}
\hline \multicolumn{6}{|c|}{11 de febrero $-1^{\circ}$ de abril del 2004} \\
\hline Lunes (L) & Martes (K) & Miércoles (M) & Jueves (J) & Viernes (V) & Sábado (S) \\
\hline 9 & 10 & 11 & 12 & 13 & 14 \\
\hline 16 & 17 & 18 & 19 & 20 & 21 \\
\hline 23 & 24 & 25 & 26 & 27 & 28 \\
\hline 1 & 2 & 3 & 4 & 5 & 6 \\
\hline 8 & 9 & 10 & 11 & 12 & 13 \\
\hline 15 & 16 & 17 & 18 & 19 & 20 \\
\hline 22 & 23 & 24 & 25 & 26 & 27 \\
\hline 29 & 30 & 31 & 1 & & \\
\hline
\end{tabular}

Fuente: Elaboración propia a partir de Casetti y di Chio (1999).

CUADRO 2

Muestra por semanas escalonadas: análisis según año, mes y días

\begin{tabular}{lll}
\multicolumn{3}{c}{ Noticias culturales } \\
Año & \multicolumn{1}{c}{ Mes } & \multicolumn{1}{c}{ Días } \\
2004 & Febrero & M 11, J 19 y V 27 \\
2004 & Marzo & S 6, L 8, K 16 y M 24 \\
2004 & Abril & J 1, V 9, S 17, L 19 y K 27 \\
2004 & Mayo & M 5, J 13, V 21, S 29 y L 31 \\
2004 & Junio & K 8, M 16 y J 24 \\
2004 & Julio & V 2, S 10, L 12, K 20 y M 28 \\
2004 & Agosto & J 5, V 13, S 21, L 23 y K 31 \\
2004 & Septiembre & M 8, J 16 y V 24 \\
2004 & Octubre & S 2, L 4, K 12, M 20 y J 28 \\
2004 & Noviembre & V 5, S 13, L 15 y K 23 \\
2004 & Diciembre & M 1, J 9, V 17, S 25 y L 27 \\
2005 & Enero & K 4, M 12, J 20, V 28 \\
2005 & Febrero & S 5 \\
\hline
\end{tabular}

Fuente: Elaboración propia.

seleccionado (sábado) y completar un año. En el Cuadro 1 se muestra un ejemplo del primer mes:

El análisis partiría del 11 de febrero del 2004 (M) y se continuaría con el 19 de febrero (J), el 27 de febrero (V), el 6 de marzo (S), el 8 de marzo (L), el 16 de marzo (K), el 24 de marzo (M) y el $1^{\circ}$ de abril (J). Así se proseguiría con los días seleccionados para la muestra, hasta llegar al 5 de febrero del 2005 (S), fecha más próxima al cierre de la periodización determinada: 10 de febrero del 2005. La muestra por semanas escalonadas quedaría definida como se muestra en el Cuadro 2.

La selección de esta técnica respondería a la posibilidad de confiar en la representatividad de la muestra: se puede analizar su comportamiento en cada uno de los meses y los días que compondrían el estudio; de ese modo, se obtendrían características confiables y particulares de las diferentes notas culturales durante un año calendario.

\section{Definición de variables para el análisis}

Para comprender mejor el proceso de recolección de datos, se presenta un cuadro de variables (Cuadro 3).

\section{Plan de recolección de los datos}

Una vez que existe certeza sobre el problema de investigación, el diseño metodológico, la población y la muestra, se trazaría el plan de acción que se seguirá para recolectar los datos. Este plan se puede esbozar a partir de las definiciones instrumentales del cuadro 3; precisamente, las condiciones instrumentales son todos aquellos recursos que coadyuvan al proceso de investigación. 
CUADRO 3

Cuadro de variables

\begin{tabular}{|c|c|c|c|}
\hline Variable & $\begin{array}{l}\text { Definición } \\
\text { conceptual }\end{array}$ & $\begin{array}{l}\text { Definición } \\
\text { operacional }\end{array}$ & $\begin{array}{l}\text { Definición } \\
\text { instrumental }\end{array}$ \\
\hline Fecha & $\begin{array}{l}\text { La fecha es la indicación del } \\
\text { lugar y tiempo. Tiempo en } \\
\text { que ocurre o se hace algo. }\end{array}$ & $\begin{array}{l}\text { La fecha es el día en que se publicó la } \\
\text { nota, entre el } 11 \text { de febrero del } 2004 \text { y el } \\
10 \text { de febrero del } 2005 \text {. }\end{array}$ & $\begin{array}{l}\text { Revisión } \\
\text { hemerográfica de } \\
\text { fuentes primarias }\end{array}$ \\
\hline Orden & $\begin{array}{l}\text { El orden es la colocación } \\
\text { de las cosas en el lugar que } \\
\text { les corresponde. Serie o } \\
\text { sucesión de las cosas. }\end{array}$ & $\begin{array}{l}\text { El orden es la disposición sucesiva con } \\
\text { que se publican las notas culturales en } \\
\text { una edición. }\end{array}$ & $\begin{array}{l}\text { Revisión } \\
\text { hemerográfica de } \\
\text { fuentes primarias }\end{array}$ \\
\hline Espacio & $\begin{array}{l}\text { El espacio es la extensión } \\
\text { que contiene toda la materia } \\
\text { existente. Es la parte que } \\
\text { ocupa cada objeto sensible. }\end{array}$ & $\begin{array}{l}\text { El espacio es una escala porcentual, en } \\
\text { que } 100 \% \text { es } 1 \text { página. Entonces, } 200 \% \\
=2 \text { páginas; } 150 \%=\text { página y media; } \\
125 \%=\text { página y un cuarto; } 75 \%=\text { tres } \\
\text { cuartos de página; } 50 \%=\text { media página; } \\
33 \% \text {, un tercio; } 25 \% \text {, un cuarto; y } 12 \% \\
\text { un octavo de página. }\end{array}$ & $\begin{array}{l}\text { Revisión } \\
\text { hemerográfica de } \\
\text { fuentes primarias }\end{array}$ \\
\hline Área o tema & $\begin{array}{l}\text { El área o tema es el orden } \\
\text { de materia o de ideas de que } \\
\text { se trata. Campo o esfera de } \\
\text { acción. Asunto o materia } \\
\text { de un discurso; este mismo } \\
\text { asunto o materia. Unidad de } \\
\text { contenido. }\end{array}$ & $\begin{array}{l}\text { Hay ocho áreas generales: artes, } \\
\text { artes escénicas, artes plásticas, artes } \\
\text { visuales, cultura, gastronomía, lengua y } \\
\text { literatura, y patrimonio, que a su vez se } \\
\text { clasifican en diversas manifestaciones. }\end{array}$ & $\begin{array}{l}\text { Revisión } \\
\text { hemerográfica de } \\
\text { fuentes primarias y } \\
\text { análisis de contenido }\end{array}$ \\
\hline
\end{tabular}

Fuente: Elaboración propia.

La recolección de información será mediante una matriz compuesta por categoría representativas de atención y relevancia (Cuadro 4) para analizar las noticias culturales. Se recogería la información requerida sobre fecha (de publicación) y orden de rangos (posteriormente se explicará y es el único que interesa, de momento, a la presente investigación); ese mismo orden de rangos definirá la relevancia de los temas por área (cultural); tipo de cultura: de elite (C. e) o popular (C.p.); fuente; origen de la noticia: nacional (Nac.) o internacional; lugar, espacio (por página) y acontecimiento motivador (A. m.)

Para completar las variables de recolección de la muestra, se deberían revisar libros, revistas, documentos electrónicos y periódicos.

\section{CUADRO 4}

Variables para la recolección de la muestra

Nombre del periódico y la sección o el suplemento

\begin{tabular}{cccccccccc} 
Fecha & Orden & Área & C. e. & C. p. & Nac. Internacional Fuente & Lugar & Espacio & A.m. \\
\hline 1. &. &. & & & \\
\hline 2. &. & & & \\
\hline
\end{tabular}

Fuente: Elaboración propia. 
Finalmente, se caracterizaría mediante la metodología de análisis de contenido, la agenda y los temas del periodismo informativo cultural de Magazine, Abanico y Cultura durante un año, para definir la conexión intermediática de los tres medios de comunicación en esa materia.

\section{Plan de tabulación y análisis de los datos}

Los datos obtenidos serían procesados electrónicamente en un programa informático (Excel de Microsoft o IBM SPSS). La información permitiría comparar las noticias culturales publicadas en la prensa escrita en Costa Rica, a fin de determinar el grado de correlación entre sus agendas informativas y determinar el orden de rangos respectivo.

\section{Orden de rangos: rompiendo el efecto de la Reina Roja}

Una breve disquisición: en la novela del matemático, fotógrafo y escritor inglés Lewis Carroll (1832-1898), A través del espejo y lo que Alicia encontró al otro lado (1871), los habitantes del país de la Reina Roja participan en una carrera que ella organiza y deben correr lo más rápido que puedan. En ese pasaje, la Reina Roja y Alicia corren tomadas de la mano hasta que la segunda se agota. Al parar, acontece lo siguiente:

"Alicia miró alrededor suyo, sorprendida.

-Pero ¿cómo? iSi parece que hemos estado bajo este árbol todo el tiempo! ¡Todo está igual que antes!

- iPues claro que sí! - convino la Reina. ¿Y cómo iba a estar si no?

- En mi país - dijo Alicia, que todavía jadeaba un poco al hablar-, cuando se corre durante algún tiempo en una determinada dirección, se suele llegar a alguna parte.

- Tu país debe ser algo lento -comentó la Reina-. Aquí tienes que correr a toda velocidad para poder permanecer en el mismo lugar, y si quieres desplazarte a otro... ;Entonces debes correr el doble de prisa!" (Carroll, 2003: 18)

Tanto real como agobiante es la situación representada: la lucha por saber quién llega el primero; la estrategia de la Reina Roja para saber quién gana. En un entorno continuamente cambiante, en constante evolución académica y tecnológica, que nos reclama en cualquier campo y desde luego como periodistas, en las salas de redacción debemos esforzarnos constantemente $\mathrm{y}$ el doble para que nuestras notas se publiquen.

Ese esfuerzo y esa competencia diaria (modelo sociosemiótico de la comunicación y factores y determinantes de la producción de mensajes; ver Rodrigo Alsina, 1995, y Gomis, 1991 en Lizano, 2011) se refleja, en este caso, en la configuración de agenda de temas (producción noticiosa) plasmada en las secciones culturales.

La metodología del orden de rangos es una categorización que sirve para determinar el resultado de ese esfuerzo, de esa "carrera": define la importancia numérica y secuencial de los temas en cada una de las tres agendas periodísticas; al mismo tiempo, posibilita verificar el grado de afinidad entre las tres agendas informativas culturales. En los cuadros 5, 6 y 7 se detalla el ejemplo

\section{CUADRO 5}

Orden de rangos de las categorías temáticas específicas para Cultura $(L N)$

\begin{tabular}{clcc} 
Rango & \multicolumn{1}{c}{ Categoría } & Absolutos & Relativos \\
1 & Literatura & 45 & $26,47 \%$ \\
2 & Plástica & 38 & $22,35 \%$ \\
3 & Música & 17 & $10,00 \%$ \\
4 & Teatro & 14 & $8,23 \%$ \\
5 & Danza & 11 & $6,47 \%$ \\
6 & Arquitectura & 7 & $4,11 \%$ \\
7 & Lengua & 6 & $3,53 \%$ \\
8 & Ópera & 4 & $2,35 \%$ \\
9 & Escultura & 3 & $1,76 \%$ \\
10 & Patrimonio & 3 & $1,76 \%$ \\
11 & Arqueología & 2 & $1,17 \%$ \\
12 & Arte contemporáneo & 2 & $1,17 \%$ \\
13 & Fotografía & 2 & $1,17 \%$ \\
14 & Cultura & 2 & $1,17 \%$ \\
15 & Cine & 1 & $0,59 \%$ \\
16 & Gastronomía & 0 & $0 \%$ \\
17 & Historia & 0 & $0 \%$ \\
- & Varios & 13 & $7,64 \%$ \\
& Total & 170 & $100 \%$ \\
\hline
\end{tabular}

Fuente: Cultura, del 11 de febrero del 2004 al 10 febrero del 2005. 
CUADRO 6

Orden de rangos de las categorías temáticas específicas para Abanico $(P L)$

\begin{tabular}{clcc} 
Rango & \multicolumn{1}{c}{ Categoría } & Absolutos & Relativos \\
1 & Música & 27 & $16,26 \%$ \\
2 & Literatura & 25 & $15,06 \%$ \\
3 & Cine & 13 & $7,83 \%$ \\
4 & Teatro & 13 & $7,83 \%$ \\
5 & Danza & 11 & $6,62 \%$ \\
6 & Plástica & 11 & $6,62 \%$ \\
7 & Arqueología & 7 & $4,21 \%$ \\
8 & Arquitectura & 5 & $3,01 \%$ \\
9 & Historia & 5 & $3,01 \%$ \\
10 & Arte contemporáneo & 3 & $1,80 \%$ \\
11 & Escultura & 3 & $1,80 \%$ \\
12 & Gastronomía & 3 & $1,80 \%$ \\
13 & Lengua & 3 & $1,80 \%$ \\
14 & Ópera & 3 & $1,80 \%$ \\
15 & Patrimonio & 3 & $1,80 \%$ \\
16 & Fotografía & 1 & $0,60 \%$ \\
17 & Cultura & 0 & $0 \%$ \\
- & Varios & 30 & $18,07 \%$ \\
& & 166 & $100 \%$ \\
\hline
\end{tabular}

Fuente: Abanico, del 11 febrero del 2004 al 10 febrero del 2005.

de orden de rangos para las categorías temáticas específicas de cada agenda:

Ahora, si se suman las tres agendas para definir el orden de rangos en la agenda nacional $(L N+L R+P L)$ se establece el orden de rangos global, compuesto por las tres agendas culturales analizadas (cuadro 8). Esto servirá para conocer cuáles son los temas culturales específicos de la prensa informativa cultural en Costa Rica, a la vez que se conocerá si algún medio marca la agenda.

En el cuadro 8 se confirma que ningún medio de comunicación escrito marca la pauta en la definición de temas: los mismos 5 o 6 son recurrentes en la agenda global: literatura, música, plástica, teatro y danza, al igual que cine.

\section{Gráficos de dispersión}

Los gráficos de dispersión son líneas de puntos que establecen la correlación entre dos variables, a partir del orden de rangos de cada una. $\mathrm{O}$ sea, estos resultados permitirían determinar
CUADRO 7

Orden de rangos de las categorías temáticas específicas para Magazine $(L R)$

\begin{tabular}{clcc} 
Rango & \multicolumn{1}{c}{ Categoría } & Absolutos & Relativos \\
1 & Música & 17 & $17,17 \%$ \\
2 & Cine & 10 & $10,10 \%$ \\
3 & Literatura & 10 & $10,10 \%$ \\
4 & Plástica & 9 & $9,10 \%$ \\
5 & Teatro & 8 & $8,08 \%$ \\
6 & Danza & 7 & $7,07 \%$ \\
7 & Cultura & 5 & $5,05 \%$ \\
8 & Escultura & 4 & $4,04 \%$ \\
9 & Arquitectura & 3 & $3,03 \%$ \\
10 & Fotografía & 3 & $3,03 \%$ \\
11 & Gastronomía & 2 & $2,02 \%$ \\
12 & Historia & 2 & $2,02 \%$ \\
13 & Ópera & 2 & $2,02 \%$ \\
14 & Arqueología & 1 & $1,01 \%$ \\
15 & Arte contemporáneo & 1 & $1,01 \%$ \\
16 & Lengua & 1 & $1,01 \%$ \\
17 & Patrimonio & 1 & $1,01 \%$ \\
- & Varios & 13 & $15,15 \%$ \\
& Total & 99 & $100 \%$ \\
\hline
\end{tabular}

Fuente: Abanico, del 11 febrero del 2004 al 10 febrero del 2005.

\section{CUADRO 8}

Orden de rangos de las categorías temáticas para la agenda nacional $(L N+L R+P L)$

\begin{tabular}{clcc} 
Rango & \multicolumn{1}{c}{ Categoría } & Absolutos & Relativos \\
1 & Literatura & 80 & $18,39 \%$ \\
2 & Música & 61 & $14,02 \%$ \\
3 & Plástica & 58 & $13,34 \%$ \\
4 & Teatro & 35 & $8,05 \%$ \\
5 & Danza & 29 & $6,67 \%$ \\
6 & Cine & 24 & $5,52 \%$ \\
7 & Arquitectura & 15 & $3,45 \%$ \\
8 & Arqueología & 10 & $2,30 \%$ \\
9 & Escultura & 10 & $2,30 \%$ \\
10 & Lengua & 10 & $2,30 \%$ \\
11 & Ópera & 9 & $2,07 \%$ \\
12 & Cultura & 7 & $1,60 \%$ \\
13 & Historia & 7 & $1,60 \%$ \\
14 & Patrimonio & 7 & $1,60 \%$ \\
15 & Arte contemporáneo & 6 & $1,38 \%$ \\
16 & Fotografía & 6 & $1,38 \%$ \\
17 & Gastronomía & 5 & $1,15 \%$ \\
- & Varios & 56 & $12,88 \%$ \\
& Total & 435 & $100 \%$
\end{tabular}

Fuente: Elaboración propia a partir de los cuadros 5 y 6. 
estadísticamente el coeficiente de correlación entre pares de agendas y, de modo complementario, permite la elaboración de gráficos de dispersión, que también funcionan con dos variables.

Cada punto en el gráfico indica la unión de los dos órdenes de rangos (del eje $X$ y del $Y$ ) para un solo tema. Ambos ejes son independientes: no son causales ni tampoco concurrentes pues tienen cualidades o circunstancias productivas distintas; o sea, estadísticamente, la tendencia temática de una agenda no incide en la otra.

Por ejemplo, si el tema teatro ocupa el puesto 2 en la primera agenda $(X)$ y ocupa el puesto 3 en la segunda agenda $(Y)$, entonces el punto en el gráfico de dispersión estará en la coordenada $(1,3)$. Del mismo modo, todos los temas de una agenda se colocan en el eje $X$ y todos los temas de la otra agenda, en el eje $Y$; Los cruces de ambas coordenadas determinan los puntos del gráfico y formarán una secuencia, en el mejor de los casos, lineal; en el peor de los casos, una nube de puntos dispersos.

$\mathrm{Al}$ graficar las dos agendas (que se pueden corroborar, en primera instancia, mediante los puntos de dispersión), se determina el grado de coincidencia, que varía entre -1 y +1 . De acuerdo con la ubicación de los puntos en el gráfico, se establece el grado de correlación: si la línea de puntos forma o se aproxima a un ángulo de $45^{\circ}$ desde el vértice del gráfico (unión de ejes $X$ y $Y$ ), la correlación de temas será mayor; pero si los puntos se dispersan, entonces un tema específico tiene un diferente orden de rango en cada una de las dos agendas comparadas. Véase la figura 1.

Con los órdenes de rangos de Cultura y Abanico, se concreta el gráfico de dispersión (Figura 2).

La figura 3 representa la dispersión para correlacionar Cultura y Magazine.

La figura 4 muestra la dispersión para correlacionar Abanico y Magazine.

\section{Coeficiente de correlación de Spearman}

Finalmente, con esos datos, se calcula el coeficiente de correlación lineal de rangos $\left(r_{s}\right) \mathrm{o}$ coeficiente de correlación de Spearman, $\mathrm{r}$ (rho), una fórmula que mide la asociación entre dos variables; se grafican dos de las agendas y se pueden corroborar, en segunda instancia, mediante tal ecuación). Entonces, se agrupan las agendas periodísticas en parejas: Cultura $(L N)$ y Abanico $(P L) ;$ Cultura $(L N)$ y Magazine $(L R) ;$ Magazine) $(L R)$ y Abanico) $(P L)$.

Este método permitiría establecer estadísticamente el nivel de relación entre dos variables independientes, que en este caso serían los temas culturales de cada sección.

Para calcular el $r_{s}$, los datos se colocan en una fórmula estadística y se reemplazan por un valor numérico. El coeficiente de correlación lineal de rangos se expresa en el cuadro 9.

CUADRO 9

Coeficiente de correlación de Spearman

$r=1-\frac{6 \sum \mathrm{D}^{2}}{\mathrm{~N}\left(\mathrm{~N}^{2}-1\right)}$

Fuente: Glass y Stanley, 1994.

donde $\mathrm{D}$ es la diferencia entre los correspondientes valores del eje de $X$ y $Y$ (cada una de las agendas), mientras que $\mathrm{N}$ es el número total de parejas de temas específicos.

Al comparar las agendas culturales de Cultura y Abanico, el coeficiente de correlación lineal de rangos $\left(r_{s}\right)$ es $+0,525$, a partir del coeficiente de Spearman, explicado en la metodología:

$$
\begin{array}{ll}
\rho=1-\frac{6 \sum \mathrm{D}^{2}}{\mathrm{~N}\left(\mathrm{~N}^{2}-1\right)} & \rho=1-\frac{2328}{4896} \\
\rho=1-\frac{6(388)}{17(289-1)} & \rho=1-0,475 \\
\rho=1-\frac{6(388)}{17(288)} & \rho=+0,525
\end{array}
$$

Es una relación moderada directa (se aproxima a $+0,50$ ): el valor de $+0,525$ es el producto del cruce de las dos agendas. 


\begin{tabular}{|c|c|c|}
\hline $\begin{array}{l}\text { Valor } \\
\text { de } r\end{array}$ & Descripción de la correlación lineal & Diagrama de dispersión \\
\hline+1 & $\begin{array}{l}\text { Relación perfecta, positiva o directa: todos los valores del } \\
\text { eje } X \text { aumentan proporcionalmente a los del eje } Y \text {. Desde el } \\
\text { vértice, la línea es de } 45^{\circ} . \text { O sea, el orden de rangos determi- } \\
\text { nó así los ejes }(X, Y):(1,1),(2,2),(3,3),(4,4) \ldots \text { Mientras más } \\
\text { se aproximen las dos agendas a esta tendencia, la correla- } \\
\text { ción temática será perfecta. }\end{array}$ &.$^{\cdot} \cdot$ \\
\hline$+0,50$ & $\begin{array}{l}\text { Relación moderada, positiva o directa: algunos valores del } \\
\text { eje } X \text { aumentan proporcionalmente a los valores del eje } Y \text {, } \\
\text { pero otros se desvían o dispersan ligeramente La relación es } \\
\text { moderada puesto que algunos temas coinciden y otros no. }\end{array}$ & 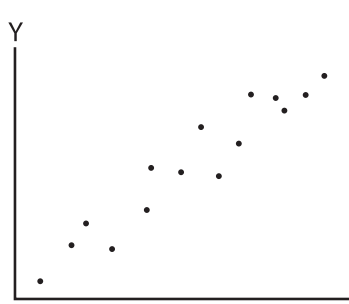 \\
\hline 0 & $\begin{array}{l}\text { Relación nula: en este caso, la nube de puntos está total- } \\
\text { mente dispersa; por lo tanto, no habría coincidencia entre } \\
\text { los temas de las dos agendas. }\end{array}$ & 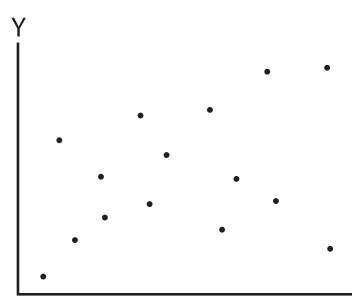 \\
\hline$-0,50$ & $\begin{array}{l}\text { Relación moderada, negativa o inversa: algunos valores del } \\
\text { eje } X \text { disminuyen en tanto los correspondientes del eje } Y \\
\text { aumentan, pero otros se desvían o dispersan ligeramente. }\end{array}$ & 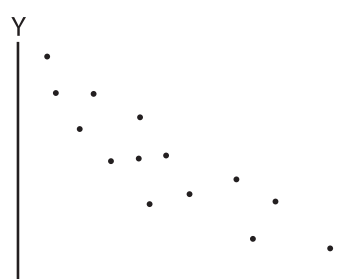 \\
\hline-1 & $\begin{array}{l}\text { Relación perfecta, negativa o inversa: todos los valores del } \\
\text { eje } X \text { disminuyen en tanto los correspondientes del eje } Y \text { au- } \\
\text { mentan; por eso es una relación perfecta pero a la inversa. } \\
\text { El orden de rangos determinó así los ejes }(X, Y):(1,7),(2,6) \text {, } \\
(3,5),(4,4),(5,3),(6,2),(7,1) \text {. En cuanto a correspondencia } \\
\text { de agendas, los casos inversos son raros. }\end{array}$ & 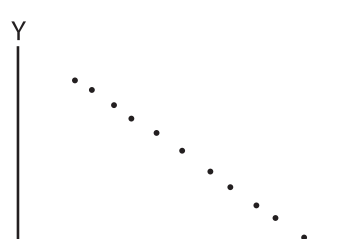 \\
\hline
\end{tabular}

Figura 1. Valor de $r_{s}$ y su gráfico de dispersión correspondiente. Fuente: Gómez (2004); Levin y Rubin (1996). 


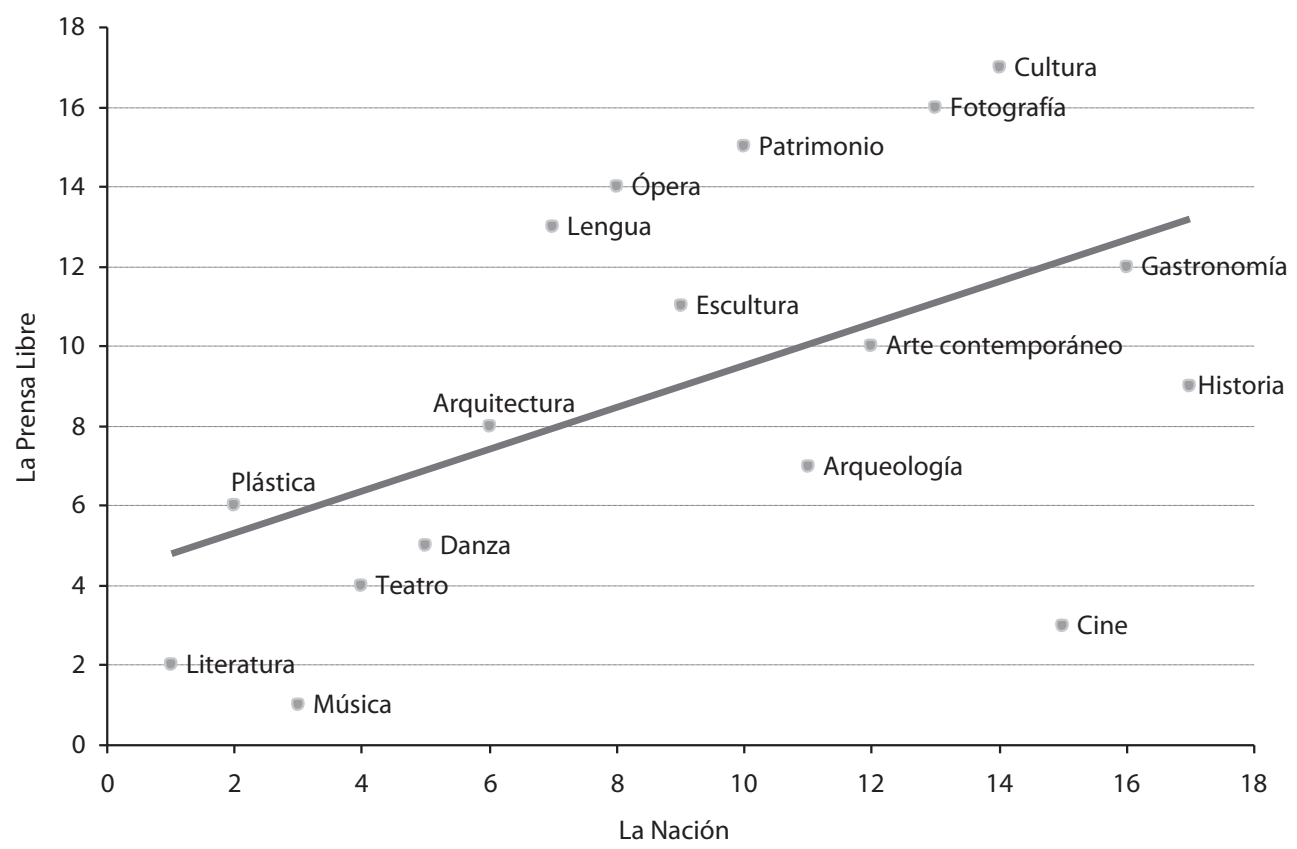

Figura 2. Gráfico de dispersión de correlación de rangos entre las agendas culturales de Cultura $(L N)$ y Abanico $(P L)$. Fuente: Elaboración propia a partir de los cuadros 5 y 6.

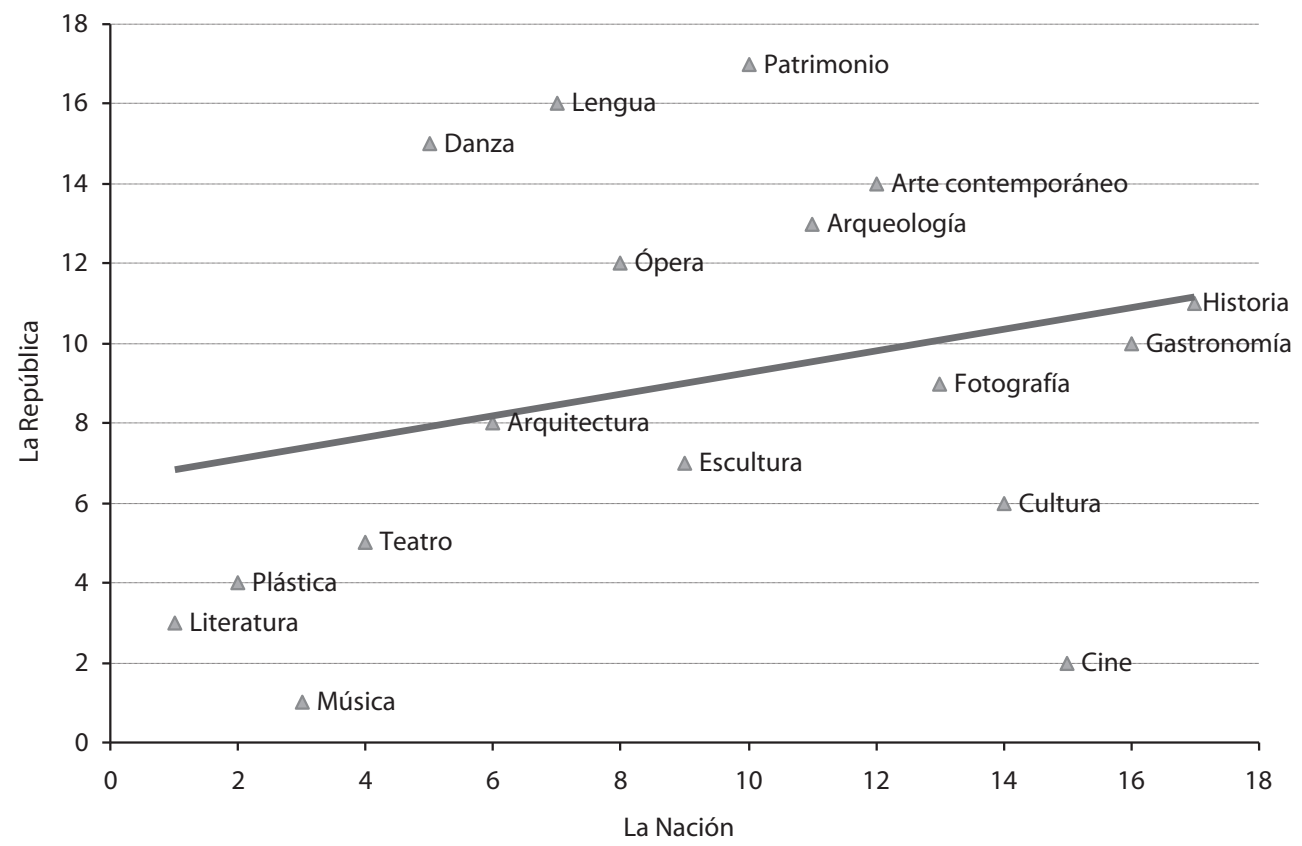

Figura 3. Gráfico de dispersión de correlación de rangos entre las agendas culturales de Cultura $(L N)$ y Magazine (LR). FUENTE: Elaboración propia a partir de los cuadros 5 y 7 . Fuente: Elaboración propia a partir de los cuadros 5 y 7. 


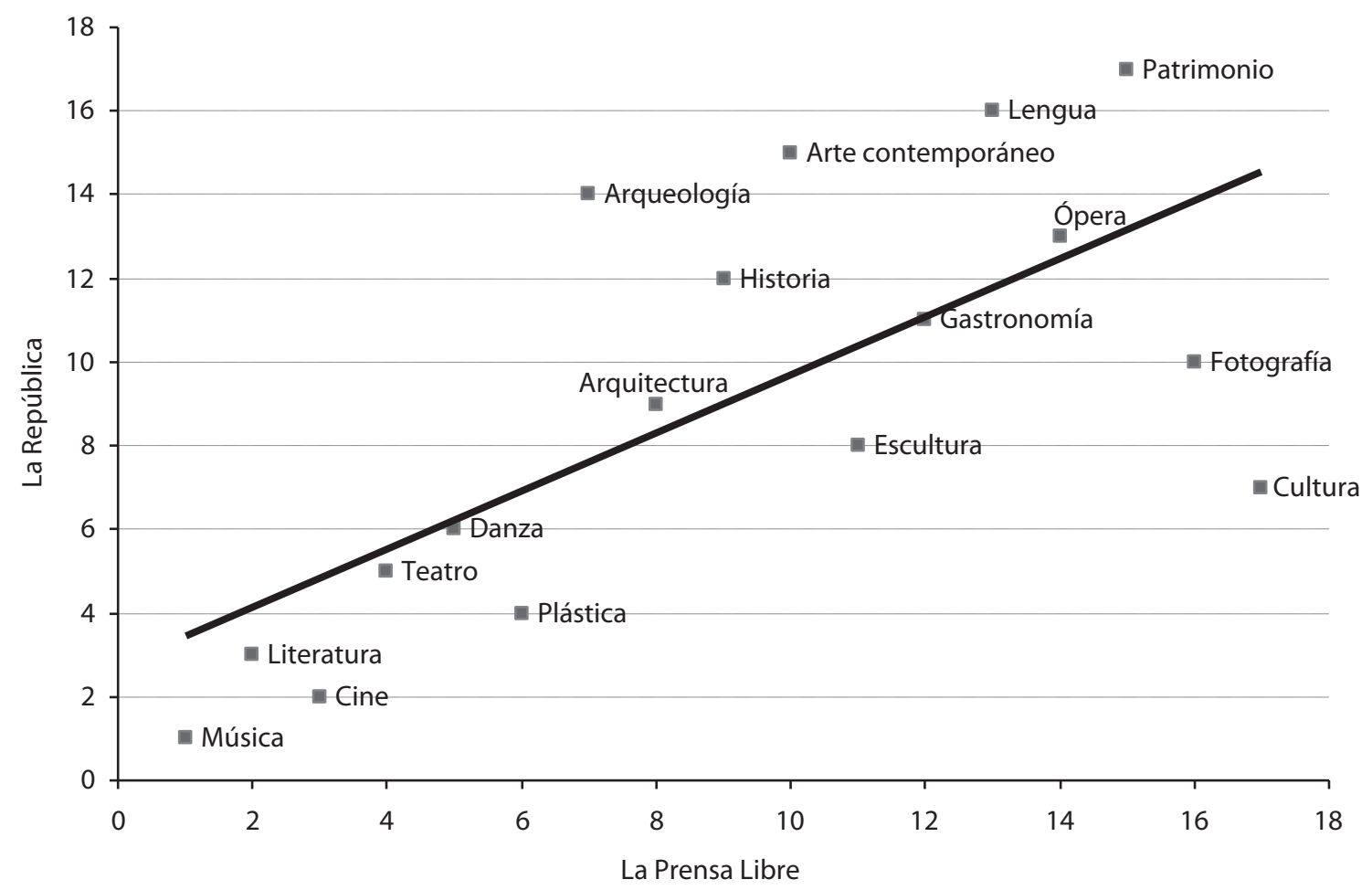

Figura 4. Gráfico de dispersión de correlación de rangos entre las agendas culturales de Abanico $(P L)$ y Magazine (LR). Fuente: Elaboración propia a partir de los cuadros 6 y 7.

Para Cultura y Magazine, el valor del $\left(r_{s}\right)$ es de $+0,429$ : como el valor tiende a $+0,50$ la relación de sus agendas es moderada directa.

$$
\begin{array}{ll}
\rho=1-\frac{6 \sum \mathrm{D}^{2}}{\mathrm{~N}\left(\mathrm{~N}^{2}-1\right)} & \rho=1-\frac{2796}{4896} \\
\rho=1-\frac{6(466)}{17(289-1)} & \rho=1-0,571 \\
\rho=1-\frac{6(591)}{17(288)} & \rho=+0,429
\end{array}
$$

El valor de $r_{s}$ para Abanico y Magazine es $+0,691$. Estos dos periódicos tienen una correlación más próxima que los otros dos casos. El número $+0,691$ se aproxima a $+0,75$ que equivale a una relación moderada:

$$
\begin{array}{ll}
\rho=1-\frac{6 \sum \mathrm{D}^{2}}{\mathrm{~N}\left(\mathrm{~N}^{2}-1\right)} & \rho=1-\frac{1512}{4896} \\
\rho=1-\frac{6(252)}{17(289-1)} & \rho=1-0,309 \\
\rho=1-\frac{6(252)}{17(288)} & \rho=+0,691
\end{array}
$$

La relación es moderada porque la mayoría de los valores de La Prensa Libre aumentan proporcionalmente a los de La República.

El estudio estadístico presentado hasta aquí se debe complementar con alguna metodología como por ejemplo un análisis de contenido, a fin de conocer la interrelación y el grado de relevancia de los temas informativos en cada agenda mediática y también comparativamente entre 
los tres periódicos impresos, pero ese es tema de otra investigación.

\section{Conclusiones}

De modo general, se concluye que solo $L a$ Prensa Libre y La República tienen un grado de correlación moderado entre sus agendas. En los otros casos, no existe correlación. Los tres periódicos mantienen un consenso implícito sobre los temas culturales; pero por la diferencia en el orden en que estos aparecen en las tres agendas. Se concluye, asimismo, que ninguno de estos medios impresos, durante el periodo estudiado, marca la agenda cultural nacional.

En este artículo descriptivo, la metodología propuesta rescata la importancia del uso de datos estadísticos en el campo de las ciencias sociales, en este caso, comunicación y periodismo. La vinculación estrecha entre la estadística y la investigación académica en ciencias sociales contribuye al estudio de esta materia en cuanto a métodos de investigación y diseños de investigación, así como la evaluación y el diagnóstico de los fenómenos sociales.

El coeficiente de correlación de rangos (la "estrategia" de la Reina Roja) es útil por cuanto expresa en qué grado (mayor, menor o medio) las agendas temáticas (o cualquier otro elemento $u$ objeto en estudio) se encuentran ordenadas, de la misma manera, en dos variables simultáneamente (X y $Y$ ). La ventaja de los coeficientes de relación es que, según Morales, (2008: 43) "se puede interpretar sin entender por qué o cómo cuantifica el grado de relación; sin embargo, es fácil entenderlo y ayuda a la interpretación porque pone de relieve la importancia de las diferencias".

Se comprueba que la metodología aplicada es teóricamente válida y proporciona resultados coherentes y estadísticamente significativos; por ende, se insta a la comunidad de investigadores en periodismo y comunicación) a que se evalúe su aplicabilidad a otros campos de la Información Periodística Especializada (IPE), como pueden ser el periodismo económico, el deportivo, o bien el de temas judiciales, políticos o servicios públicos, por citar algunos casos.

Un enfoque estadístico es una de las muchas posibilidades para aprovechar las herramientas de las ciencias exactas en los estudios en la rama de las ciencias sociales, sin que signifique que estas pierdan su rasgo humanista, en tanto representan fenómenos de la realidad circundante. A partir de la propuesta detallada, deberá surgir un proceso de realimentación en la comunidad de investigadores para aceptar o no el modelo metodológico planteado.

\section{Bibliografía}

Carroll, Lewis (2003). A través del espejo y lo que Alicia encontró al otro lado. Buenos Aires: Biblioteca Virtual Universal. Recuperado de http://www.biblioteca.org.ar/ libros/11391.pdf

Casetti, Francesco; Di Chio, Federico (1999). Análisis de la televisión. Barcelona: Ediciones Paidós Ibérica, S.A.

Glass, G.; Stanley, J. S. (1994). Métodos estadísticos aplicados a las ciencias sociales. México: Prentice-Hall Hispanoamericana.

Gómez, M. (2004). Elementos de estadística descriptiva. San José: Editorial de la Universidad Estatal a Distancia (EUNED).

Hansen, Anders; Simon Cottle, Ralph Negrine y Chris Newbold (2002). Mass communication research methods. Londres: MacMillan.

Hernández, Roberto; Fernández, Carlos; Baptista, Pilar (2006). Metodología de la investigación. ( $4^{\mathrm{a}}$ ed.). México: McGraw Hill / Interamericana.

Levin, Richard y David Rubin (1996). Estadistica para administradores. Sexta edición. México: Prentice Hall.

Lizano, Alejandro (2011, mayo). Descifrando el Jabberwocky: definición del periodismo informativo cultural en Costa Rica. V Jornadas de Investigación: Bifurcaciones de la comunicación social. San José: Escuela de Ciencias de la Comunicación Colectiva, Universidad de Costa Rica.

Morales, Pedro (2008). Correlación y covarianza. Estadística aplicada a las Ciencias Sociales. Madrid: Universidad Pontificia Comillas, 1-48. Recuperado de http://www. upcomillas.es/personal/peter/estadisticabasica/Correlacion.pdf 
Sáenz, Randall (1999). Agenda editorial de los medios costarricenses de comunicación escrita y su relación con la agenda pública. Tesis para optar por el grado de Licenciatura en Ciencias de la Comunicación Colectiva con énfasis en Periodismo. San José: Universidad de Costa Rica.
Sandoval, Carlos (2002). Otros amenazantes. San José: Editorial de la Universidad de Costa Rica (EUCR).

Tamayo y Tamayo, Mario (1994). El proceso de la investigación científica. México: Limusa. 
- 